\title{
La ressource patrimoniale, outil de diversification touristique? Le pôle d'excellence rurale du Néouvielle, entre innovation et recyclage
}

\author{
Jacinthe Bessière ${ }^{1}$, Sylvie Clarimont ${ }^{2}$, Vincent Vlès ${ }^{3}$ \\ 1 Sociologie, Université Toulouse-Jean Jaurès, UMR5044 CERTOP, 31058 Toulouse Cedex 9, France \\ 2 Géographie, Université de Pau et des Pays de l'Adour, UMR5319 PASSAGES, 64000 Pau, France \\ 3 Urbanisme, Université Toulouse-Jean Jaurès, UMR5044 CERTOP, 31058 Toulouse Cedex 9, France
}

Au moins en France, les politiques de protection et de valorisation du patrimoine naturel ont toujours été historiquement marquées par l'ambivalence - comme en témoigne l'exemple des parcs nationaux censés concilier conservation de la nature, accueil touristique et développement de zones rurales fragiles. C'est ce type de compromis qu'aborde également l'article qui suit - dans un contexte qui n'est plus celui des années 1960, mais qui est aujourd'hui marqué par le développement durable, la transition écologique et l'injonction à l'innovation ou à l'excellence locale. Analysant de l'intérieur le projet « Néouvielle, destination nature »-construit en principe autour de la mise en valeur de ce patrimoine pyrénéen -, il montre la difficulté à inventer des formes réellement innovantes d'articulation entre protection de la nature et développement local ; et finalement à dépasser un usage purement rhétorique de la valorisation patrimoniale. Cela illustre aussi la distance qui peut exister entre la communication sur la nature et l'intégration des réalités écologiques dans les politiques locales.

La Rédaction

\section{Mots-clés :}

territoire ;

gouvernance ;

patrimoine ; diversification ; tourisme

\begin{abstract}
Résumé - Face au renouvellement des pratiques dans le domaine du tourisme et à l'évolution des territoires de montagne, la diversification touristique devient un enjeu central. Le processus de valorisation des ressources patrimoniales apparaît dès lors comme un levier de développement territorial pour lutter contre un modèle « tout ski » monospécialisé. En s'appuyant sur l'analyse d'un dispositif d'excellence et d'innovation territoriale, le Pôle d'excellence rurale, mis en œuvre en 2011 dans le massif du Néouvielle (Pyrénées), l'article questionne le patrimoine comme ressource au service d'un nouveau système de production touristique ; il examine notamment, à travers les logiques de gouvernance et les jeux d'acteurs qui en découlent, les formes de régulation qui ont abouti à un processus de détournement de l'objectif de valorisation patrimoniale, rendant illusoire, dans le cas étudié, la stratégie de diversification touristique.
\end{abstract}

\section{Keywords:}

territory; governance; heritage; diversification; tourism

\begin{abstract}
Heritage resources: a tool for tourism diversification? The Neouvielle Pôle d'Excellence Rurale scheme: innovation or recycling of old ideas. Faced with new touristic practices, the evolution of public structures and the (re)structuring of mountain territories, a key issue for development rests on tourism diversification and, presumably, reorganisation of touristic areas. Considered for decades for their sole touristic and leisure vocation, mountain territories are nowadays the focus of decisions to reorganise their socio-economic, environmental and cultural sectors. The existing mono-centred production model, focussing on a single type of activity, often at a single site is being questioned. In this context the valorisation process of heritage resources often appears as a key pointer in analyses dealing with a territorial approach to territory development. Based on the analysis of a series of decisions regarding territorial excellence and innovation - the Pôle d'Excellence Rurale (PER) set up in 2011 in the Neouvielle massif, French Pyrenees - the paper questions the potential of heritage considered as a resource to foster
\end{abstract}

Auteur correspondant : J. Bessière, bessiere@univ-tlse2.fr 
a new tourism production system for mountain territories. From a detailed qualitative survey of governance logics and the resulting interactions between the players involved, the paper highlights the various types of regulations which have led to diverting the heritage valorisation objective, thus making any attempt at a tourism diversification strategy illusory.

Ces quarante dernières années, l'économie touristique a porté le développement de la montagne. Atout France ${ }^{1}$ (2013) rappelle que $55 \%$ des investissements touristiques et $15 \%$ du chiffre d'affaires touristiques français sont réalisés en montagne. Le tourisme représente 100000 emplois salariés, soit 7,8\% du total de l'emploi salarié dans les Alpes et 12,3 \% dans les Pyrénées. Dans ce massif, une bonne vingtaine de stations se trouvent en situation de fragilité en raison d'une concurrence croissante, dans un contexte de marché européen mature, et de leur faible performance environnementale du fait de la réallocation courante $\mathrm{du}$ foncier au détriment des espaces agricoles et naturels. Leur situation se caractérise par un domaine skiable réduit, des équipements vieillissants, une capacité faible des hébergements commercialisables, un niveau de services insuffisant pour les clientèles internationales et un champ commercial réduit (Prats et al., 2000). Cette situation les pousse à rechercher un modèle d'adaptation à une forme nouvelle de modernité qui n'est plus celle du « tout ski » (Bourdeau, 2009; Clarimont et Vlès, 2008). Une recherche ${ }^{2}$ menée dans les stations de montagne pyrénéennes sur ce sujet a permis d'apporter des réponses à ces questions sous deux angles : celui des mutations socioenvironnementales comme facteurs $\mathrm{d}$ 'adaptation et celui de la diversification d'activité envisagée comme un résultat de la prise en compte, pour ces installations, des mutations à venir.

Dans cette démarche, la patrimonialisation, entendue comme un processus de construction et de valorisation sélective des patrimoines (Bessière, 2001 ; Micoud, 2004), renvoie à des logiques de mobilisation d'acteurs, autour de ressources, au service de projets de territoire. Convoqué pour accroître ou améliorer la production touristique locale, ce processus s'inscrit indéniablement dans les stratégies d'innovation (Bessière, 2012) et de mutation de territoires qui utilisent le patrimoine comme levier de projet ou pour marquer une identité.

Par cette recherche, nous avons voulu comprendre comment le patrimoine, dans sa diversité, pouvait « faire

\footnotetext{
1 Atout France est l'agence partenariale de développement touristique de l'État français qui regroupe, sous la forme juridique d'un groupement d'intérêt public, les services officiels d'étude, d'ingénierie et de représentation de la France à l'étranger, à l'image de VisitBritain en Grande-Bretagne, de l'ENIT en Italie ou de VisitSweden en Suède.

2 Recherche entreprise par les auteurs dans le cadre du programme « Les trajectoires des aires touristiques dans le Grand Sud-Ouest français » (TRATSO) (Vlès, 2015).
}

ressource » au service d'un nouveau système de production dans les territoires de montagne; en quoi il était susceptible de réguler la place des différents secteurs d'activité, quelle était sa capacité à porter de l'innovation, à être fédérateur et structurant dans des aires touristiques en quête de diversification et d'élargissement de leurs ancrages initiaux ${ }^{3}$. Le cas du pôle d'excellence rurale (PER) de Néouvielle a été choisi, car ses acteurs affichaient résolument dans leur projet le développement $\mathrm{du}$ « tourisme durable d'altitude » et la « valorisation du patrimoine ». Nous nous sommes ainsi donné pour objectif de comprendre les types de ressources patrimoniales revendiquées par les acteurs de ce territoire afin de déceler d'éventuels nouveaux modes de fonctionnement permettant de répondre aux défis de la transition touristique, entendue comme transformation lente de la sphère sociale de la production et de la consommation touristiques mondiales. Les recompositions socioculturelles et économiques induites peuvent s'accompagner de tensions territoriales et de conflits entre tourisme et problématiques environnementales et patrimoniales (Clarimont et Vlès, 2016). La notion de transition touristique est donc à prendre non seulement dans sa dimension empirique de cadre et d'objet d'action publique, mais également comme révélatrice d'un changement de statut des rapports nature/culture/société dans les territoires touristiques contemporains. Pour les auteurs, cette dimension n'est pas réductible à des décisions managériales ou politico-administratives tenant mieux compte de la préservation de l'environnement ou de la valorisation patrimoniale, ni à des formes de la contestation civique des aménagements touristiques programmés. Elle inscrit plus largement le tourisme dans une transition globale (écologique, mais aussi de la société) de transformation des rapports des sociétés occidentales à la nature et au patrimoine. Le PER Néouvielle estil porteur d'une telle dynamique de changement?

Les élus du massif, en s'engageant dans ce dispositif, ont déclaré faire de la ressource patrimoniale un vecteur de développement durable censé répondre à la fois aux défis du changement climatique, du renouvellement des populations, de l'identité des territoires ou du changement des comportements des clientèles. Ainsi, au-delà

3 La notion d'ancrage renvoie à des questions d'adéquation de la station au sein de son système productif, à la territorialisation d'une activité économique en termes de ressources (Mollard et Pecqueur, 2007 ; George-Marcelpoil, 2007 ; Vlès, 2014). 
des effets d'affichage, quelle est la réalité du changement de segment touristique promis par les porteurs de projet ?

Pour le déterminer, nous avons d'abord examiné la documentation technique et réglementaire. Nous avons ensuite mené des entretiens semi-directifs auprès de 18 acteurs ${ }^{4}$ impliqués dans la valorisation des ressources patrimoniales du territoire du Néouvielle. Nous avons également élaboré des diagnostics touristiques des formes patrimoniales du Néouvielle et étudié la mise en place de paniers de biens patrimoniaux (Pecqueur, 2001). Ces travaux s'inscrivent dans une recherche plus large (Programme TRATSO, voir aussi note 4) qui étudie comment le patrimoine peut faire « ressource » aujourd'hui dans ces territoires de montagne, quelle est sa place dans les processus de diversification touristique et quels sont les facteurs de blocage qu'il convient de dépasser.

La première partie de l'article expose la variabilité et la mise en tension de ces logiques dans la mise en œuvre de la politique $d^{\prime}$ ' excellence territoriale », en examinant les formes de pilotage engagées sur le territoire du Néouvielle. La deuxième s'attache à analyser les jeux $\mathrm{d}^{\prime}$ acteurs qui ont surtout suscité des effets d'opportunité et une dynamique cloisonnée, finalement peu ouverte à l'innovation. Enfin, la dernière partie explique comment ces formes de régulation ont abouti à un processus de détournement de l'objectif de valorisation patrimoniale, rendant ainsi la diversification touristique, telle qu'initialement annoncée, résolument difficile.

\section{La politique des pôles d'excellence rurale : d'une logique de zonage à une logique de distinction}

La politique des pôles d'excellence rurale (PER) a été mise en œuvre par l'État à partir de 2005. Elle s'inscrit dans le prolongement de la politique des pôles de compétitivité et vise, à la suite d'une procédure d'appel à projets, à distinguer, par l'octroi d'un label, des territoires ruraux porteurs d'un « projet innovant » et méritant à ce titre d'être aidés financièrement. Elle encourage la cohérence des stratégies autour de projets pilotes fédérateurs. En introduisant des thèmes cibles, elle promeut l'expérimentation et la recherche d'actions de développement novatrices. Ainsi les PER sont-ils représentatifs de la transformation des politiques en faveur du développement territorial et de l'évolution des fonctions des espaces ruraux dans l'économie et la société globales (Barthe et al., 2009). En renouant un lien direct avec les

\footnotetext{
4 Les acteurs interrogés comprennent des élus, des responsables associatifs, des acteurs institutionnels et socioprofessionnels. La liste des acteurs figure dans le rapport du programme de recherche TRATSO (Vlès, 2015).
}

territoires ruraux et de montagne et en prônant la primauté de l'« excellence », cette politique permet à l'État de « reprendre la main » sur les processus de développement local qu'il avait décentralisés à partir de 1982.

\section{L'excellence territoriale : une politique de rupture}

$L^{\prime}$ « excellence territoriale » s'inscrit en rupture avec la logique de zonage, mode d'intervention privilégié en faveur de l'espace rural depuis les années 1960. Issu du «zoning » anglo-saxon, le zonage, défini sur la base de critères statistiques (taux de chômage, taux de population agricole, densité de population, etc.), a longtemps été la forme quasi exclusive de territorialisation de l'action publique grâce à des dispositifs incitatifs (aides et exonérations diverses). Des zones spéciales d'action rurale (loi $\mathrm{n}^{\circ} 60-808 \mathrm{du} 5$ août 1960 d'orientation agricole) aux zones de rénovation rurale de 1967, cette logique fut largement reprise par les collectivités territoriales dans leurs schémas d'intervention. En raison de la raréfaction de l'argent public, cette logique de répartition des fonds en fonction des besoins des territoires est peu à peu entrée en crise. Au début des années 2000, les limites de ce type d'intervention publique sont amplement soulignées par l'État : empilement de zonages peu lisibles, rigidité de certains périmètres, critères d'éligibilité parfois contestables et effets des dispositifs incitatifs difficilement appréciables (Siné, 2001). Avec l'affirmation du néolibéralisme et de son corollaire, la « compétitivité des territoires », $l^{\prime}$ « excellence territoriale » s'impose alors comme une heureuse alternative. Cette politique dite $\mathrm{d}^{\prime}$ « excellence » concrétise le glissement du principe de solidarité entre territoires (qui prévalait dans la politique par zonages d'intervention) à celui de compétitivité territoriale, érigé en nouveau paradigme de l'action. Elle prétend mettre en mouvement les territoires ruraux en les incitant à mobiliser leurs "potentiels de compétitivité » dans une logique distinctive (Alvergne et De Roo, 2008). En effet, la politique des pôles d'excellence rurale, lancée en décembre 2005, est l'équivalent pour les territoires ruraux ou de montagne de la politique des pôles de compétitivité initiée en 2004 pour les métropoles. Même si ses ambitions sont moindres (les fonds mobilisés par l'État sont en effet bien inférieurs pour les PER que pour les pôles de compétitivité), elle s'en inspire sur le plan méthodologique (recours à une procédure d'appel à projets nationale), sur le plan de la gouvernance (incitation au partenariat entre secteur public et secteur privé) et enfin sur le plan conceptuel dans la mesure où l'injonction d'innovation y est centrale.

Dans ce cadre, deux générations d'appel à projets ont été lancées par l'État français : la première a donné lieu, en 2006, à 379 labellisations (contre 300 initialement prévues) ; la seconde, lancée en 2009, s'est soldée par 263 labellisations. Dans les deux cas, il s'est agi de 
« soutenir les dynamiques d'initiative rurale et encourager l'innovation » (PER, 2014) et plus spécifiquement de « soutenir des projets générateurs d'activité économique et de développement local en favorisant de nouvelles dynamiques territoriales, tant sur le plan organisationnel, en termes de gouvernance et de relations entre acteurs, que par les effets de levier améliorant la valorisation du potentiel local préexistant » (Ministère de l'Espace rural et de l'Aménagement du Territoire et ministère de l'Alimentation, de l'Agriculture et de la Pêche, 2010). Le PER est donc un projet de développement économique axé sur des secteurs d'activité considérés alors par la Délégation interministérielle à l'Aménagement du Territoire (Datar) comme étant caractéristiques des espaces ruraux, qu'il s'agisse d'activités productives ou de services (Lardon et al., 2014). Il est destiné à porter des projets visant la promotion des richesses naturelles, culturelles et touristiques (patrimoine), la valorisation et la gestion des ressources naturelles dans une perspective environnementale (développement durable), l'offre de services et l'accueil de nouvelles populations, l'aide au développement de productions industrielles et artisanales, de services locaux, avec en particulier l'utilisation de techniques innovantes.

\section{L'excellence territoriale et l'innovation : un nouveau référentiel pour les territoires ruraux}

Pour évaluer le projet de PER Néouvielle, nous interrogeons la notion $\mathrm{d}^{\prime}$ « excellence territoriale » mise en avant, en comparant les réalisations aux résultats attendus et à la plus-value produite dans le territoire par le dispositif. Le terme $\mathrm{d}^{\prime}$ ' excellence » désigne, dans le langage courant, la manifestation de la supériorité de quelque chose ou de quelqu'un, dans un domaine donné. Appliqué au territoire, il renverrait donc à la capacité des acteurs à se mobiliser pour mettre en œuvre un projet singulier et de qualité dans un domaine. Rarement explicitée, complexe et polysémique, la notion $\mathrm{d}^{\prime}$ " excellence territoriale » comporte au moins cinq dimensions : irrigation (retentissement d'un projet sur un territoire plus étendu que le périmètre strict de mise en œuvre), spécification (capacité à mettre en valeur les atouts du territoire), dynamique (capacité du projet à jouer un rôle de levier de développement), gouvernance et innovation (Barral et al., 2010). Notion pluridimensionnelle, $l^{\prime}$ « excellence territoriale » constitue un référentiel nouveau, élaboré au niveau national et imposé à des territoires ruraux aux moyens limités, sommés de mobiliser leur potentiel de croissance (Landel et Sénil, 2008). Dans une France de plus en plus décentralisée, l'injonction d'excellence portée par l'État témoigne d'un « gouvernement à distance » (Epstein, 2009), produisant de nouvelles normes d'action et évaluant leur bonne prise en compte locale. C'est une forme de basculement dans la vision des potentiels de développement, qui porte un regard volontairement positif sur des territoires considérés comme détenteurs de ressources spécifiques (notamment patrimoniales) et de capacités d'organisation (territoires de projets, ingénierie de développement, systèmes de gouvernance) susceptibles d'engendrer des modes de développement dynamiques. Cette notion d'excellence renvoie à celle de l'innovation et structure son dispositif politique.

L'innovation, pour sa part, désigne l'introduction d'une nouveauté dans une situation établie. Elle représente la mise en œuvre d'une invention et son intégration dans un milieu social (Alter, 2000). Ainsi, l'invention se transforme en innovation lorsque des acteurs parviennent à lui donner un sens. Elle représente, pour les entreprises et les territoires, un facteur de compétitivité et de développement, pour l'usager final un facteur d'amélioration de sa condition de vie. Dans une étude sur le système régional d'innovation (SRI) au Québec (Doloreux et al., 2005 ; Siné, 2001), les auteurs concluent que l'innovation est le résultat d'un produit social et territorialisé ; ils portent une attention particulière aux rapports entre l'entreprise innovante et les apports externes. La capacité d'innover d'un territoire dépend de son aptitude à attirer, collecter, générer, former, mais aussi à retenir et à accumuler le capital humain.

Dans le cas du dispositif PER Néouvielle, et en s'appuyant sur une méthode évaluative déjà conduite (Barthe et al., 2009), trois types d'innovation ont été recherchés : l'innovation technologique, qui désigne une avancée technique ou technologique prolongée par une application concrète et mise en œuvre dans le cadre du PER, l'innovation sociale ou organisationnelle (l'élaboration du PER et sa mise en œuvre ont-elles permis un enrichissement des rapports d'acteurs sur le territoire porteur ou une amélioration des réseaux de partenariat ?), et l'innovation institutionnelle, le dispositif PER étant censé constituer à lui seul une innovation en matière de gouvernance et d'action publique. Dans ce contexte de quête de renouvellement et de compétitivité territoriale, en quoi la ressource patrimoniale, en tant qu'outil de diversification touristique (donc de transition), constitue-t-elle une innovation permettant au PER du Néouvielle d'atteindre $\mathrm{l}^{\prime}$ « excellence » ?

\section{Le PER « Néouvielle, destination nature »: un objectif de valorisation de la ressource patrimoniale}

Le PER « Néouvielle, destination nature » appartient à la seconde génération de PER, lancée par le gouvernement en septembre 2009. Il concerne au sens strict un espace de haute montagne, le massif granitique du Néouvielle, délimité par des vallées au riche patrimoine bâti, reconnu par le label « Pays d'art et d'histoire » : la vallée d'Aure au sud et à l'est, la vallée de Barèges au 


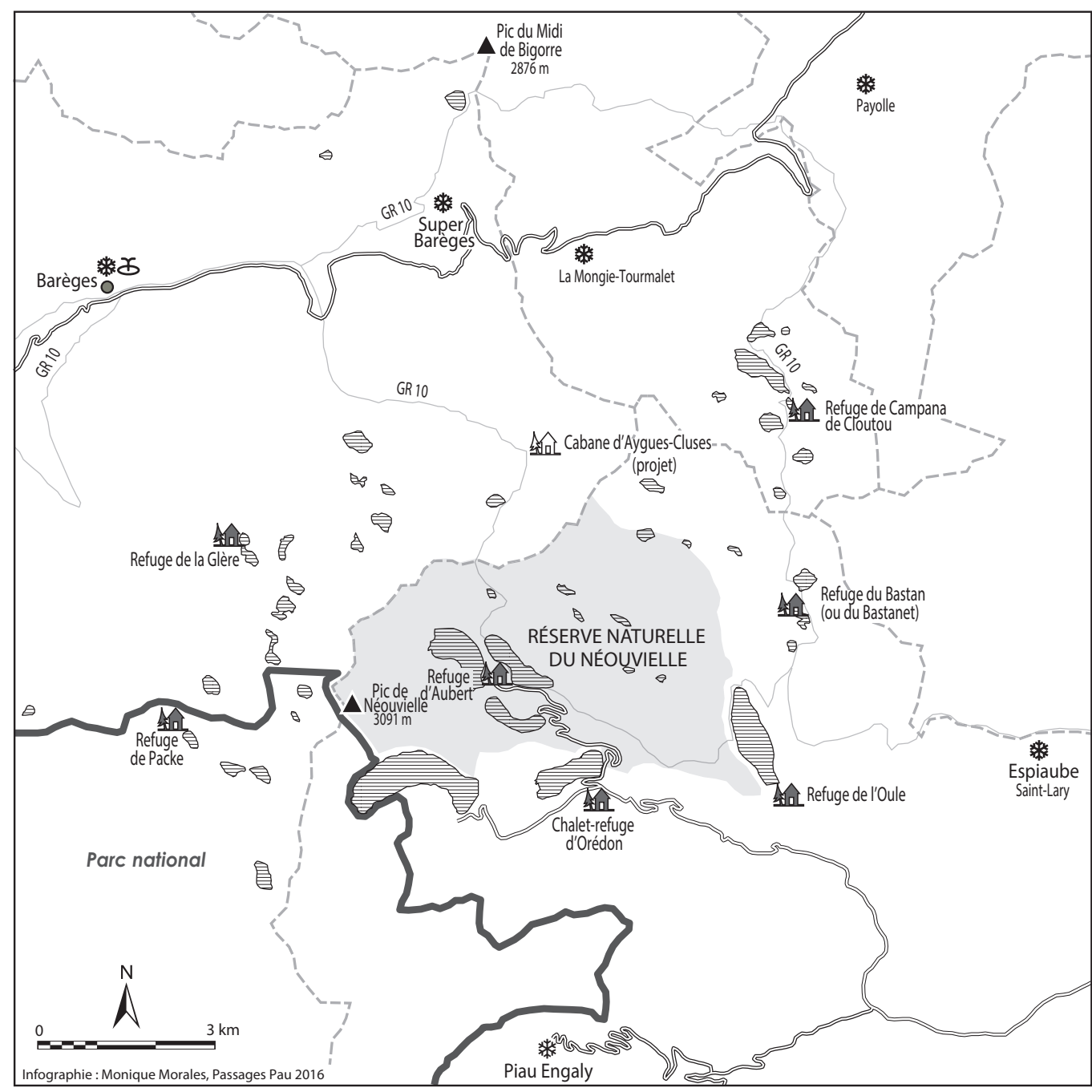

\begin{tabular}{|cl|}
\hline & Limite de la Réserve Naturelle du Néouvielle \\
\hline L & Limite du Parc National des Pyrénées \\
& Station de ski \\
& Itinéraire \\
\hline--- . Limite de canton \\
\hline & Routes départementales \\
\hline \hline
\end{tabular}

Fig. 1. Le territoire de la réserve naturelle du Néouvielle (source : V. Vlès, M. Morales).

nord-ouest, celle de Campan au nord-est avec son grand site régional pic du Midi de Bigorre, et la vallée de Gavarnie-Gèdre (inscrite au patrimoine mondial de l'Unesco) à l'ouest (fig. 1). Compris entre 1500 et $3000 \mathrm{~m}$, cet espace marqué par de nombreuses formes d'érosion et d'accumulations glaciaires offre de vastes paysages hérités de lacs et de zones humides. Il est dépourvu de toute zone d'habitat permanent et a longtemps été exclusivement dédié au pastoralisme. Pourtant, s'il est souvent décrit comme un haut lieu naturel, le massif du Néouvielle est un espace très aménagé. Les aménagements hydroélectriques réalisés en deux phases, avant la Première Guerre mondiale et après la Seconde, ont modifié considérable- ment la physionomie du massif : construction des barrages d'Orédon et de l'Oule, dès le début du XXe siècle, puis édification à $2160 \mathrm{~m}$ d'altitude du barrage de Capde-Long par EDF entre 1948 et 1953. Le développement des pratiques récréatives dès le $\mathrm{XIX}^{\mathrm{e}}$ siècle a également contribué à diversifier les usages d'un massif d'accès aisé par voie routière ou par remontée mécanique. Espace exploité et aménagé, le Néouvielle est aussi un site naturel qui bénéficie de plusieurs dispositifs de protection, souvent juxtaposés et instaurés parfois selon une logique défensive - en 1981, le classement du site de l'Oule Pichaleye a ainsi été opéré en réaction à un projet de liaison Saint-Lary/La Mongie (Clarimont et Bessière, 
2016). Le niveau de protection le plus élevé est offert par la réserve naturelle nationale ( 2300 ha gérés par le parc national depuis 1968) dont les origines remontent à 1935 avec la création d'une première réserve à l'initiative de scientifiques de la Société nationale d'acclimatation de France. Â cette réserve s'ajoutent quatre sites classés au titre de la loi de 1930 pour leur valeur paysagère : bassin du Bastan (1932), abords du col du Tourmalet (1942), nvallons d'Estibère, Gourguet et Guits (1951), l'Oule Pichaleye (1981). Le Néouvielle est également intégré au réseau Natura 2000 et jouxte le Parc national des Pyrénées.

L'intitulé du PER «Néouvielle, destination nature » s'inscrit à la croisée des deux types de dénomination les plus courants pour les PER (Landel et Sénil, 2008). Il fait à la fois référence au périmètre géographique du projet le massif du Néouvielle - et à l'horizon du projet - la construction d'un produit touristique valorisant le patrimoine naturel par la réalisation d'un « grand tour » dédié à la randonnée. Le développement d'un «tourisme durable d'altitude » constitue l'objectif prioritaire d'un projet qui s'articule autour de quatre actions : 1 . la création d'un produit « tour de massif » et la mise en réseau des refuges par un système de réservation unique en ligne ; 2. l'amélioration de l'accueil et des accès au massif avec la mise en place d'au moins six portes d'entrée réparties dans les trois vallées d'accès (reposant, en fait, sur la rénovation de parkings déjà existants avec panneaux de type "relais informations services ») ; 3 . la création de plusieurs circuits adaptés aux personnes en situation de handicap ; 4 . la modernisation, l'extension et la mise aux normes environnementales de refuges largement obsolètes (voire la construction d'un nouvel hébergement dans le site classé d'Aygues Cluses) (Convention-cadre État/Association pour la valorisation du massif du Néouvielle du 14 mars 2011). Le projet est donc principalement un projet de développement touristique via une mise en valeur du patrimoine naturel. Le montant total des investissements est estimé à 3,73 millions d'euros HT, financé par les maîtres d'ouvrage de chaque action (30\%), l'État (PER, $25 \%$ ) et des subventions des autres collectivités ( $45 \%$ ). Outre ces opérations d'équipement touristique, le PER souhaite l'instauration d'une nouvelle forme de gouvernance territoriale mixte associant étroitement acteurs publics et privés.

Le portage du projet est ainsi confié à une association, 1'Association pour la valorisation du massif du Néouvielle, créée en 2007 et présidée par la conseillère générale de Vielle-Aure ${ }^{5}$ et vice-présidente du conseil général. Cette association regroupe, outre les 14 communes concernées par le massif, les propriétaires fonciers

5 Le canton de Vielle-Aure a été supprimé par la réforme cantonale de 2014. La commune est désormais intégrée au canton Neste, Aure et Louron. (essentiellement publics), les propriétaires des refuges existants ${ }^{6}$, les gestionnaires de ces refuges, le comité départemental du tourisme des Hautes-Pyrénées, la compagnie des guides des Pyrénées, l'Association départementale des accompagnateurs de montagne des Hautes-Pyrénées, les gestionnaires d'estives, les fédérations départementales de chasse et de pêche et le parc national. Elle s'appuie sur le syndicat à vocation unique (SIVU) Aure-Néouvielle formé avec les communes d'Aragnouet (station de Piau-Engaly), Aspin-Aure, Saint-Lary-Soulan (station de ski éponyme) et VielleAure. Porté par une structure associative assez atypique, le PER Néouvielle souhaite, afin d'accéder au label, «mettre les territoires ruraux en mouvements, en renforçant l'attractivité des territoires et en répondant aux attentes des habitants en s'appuyant sur un partenariat public/privé fort » (Association pour la valorisation du massif du Néouvielle, 2010). Le PER est labellisé en août 2011 pour « son inscription rurale, la qualité du partenariat public/privé [et] sa contribution au développement durable $^{7}$ ». Affichant les principes du développement durable et de l'excellence environnementale, le projet met l'accent sur les ressources patrimoniales : patrimoine bâti et, surtout, patrimoine naturel dans le cadre des sports et activités de nature. Il est porté par une structure associative sous contrôle politique (la présidence et le bureau sont confiés à des élus), au fonctionnement souple, mais intégrant des acteurs nombreux et variés, ce qui impliquait d'emblée une gouvernance complexe.

\section{La gouvernance du PER Néouvielle : effet d'aubaine et gestion des interdépendances}

Au cours des dernières décennies, le concept de « gouvernement » en tant qu'incarnation du seul pouvoir de décision légitime a été progressivement remplacé par la notion multiscalaire, polysémique et polycentrique de " gouvernance ». Terme qui a fait l'objet de conceptualisation, la gouvernance renvoie aux transformations de l'État et à la reconfiguration de l'action publique (Le Galès, 2013). Il désigne en général un mode d'organisation du territoire alternatif au gouvernement descendant, hiérarchique et pyramidal, fondé sur des formes variées de coopération, de négociation entre acteurs publics et privés. La "gouvernance territoriale » peut ainsi être définie comme un processus complexe de coordination des acteurs - institutionnels et non institutionnels -, déployé à l'échelle locale et porteur de

\footnotetext{
6 Comme le refuge du Club alpin français (Campana de Cloutou, situé à $2225 \mathrm{~m}$ d'altitude en bordure de la réserve, sur la commune de Bagnères-de-Bigorre) ou celui de l'ASPTT de Toulouse, le refuge de Bastan, à 1351 m d'altitude, à proximité des lacs du Bastanet (commune de Saint-Lary-Soulan).

7 Décret 2011-1019 du 25 août 2011.
} 
dynamiques de « construction de la territorialité et d'appropriation des ressources » (Leloup et al., 2005). Elle prend appui sur des dispositifs, des méthodes et des outils divers qui renvoient tous à la question de la technologie et de son usage, à celle de l'apprentissage - technique, mais surtout organisationnel - induit par l'émergence de nouveaux acteurs dans la construction de projets de territoire, et également à celle de la participation (Lardon et al., 2008). L'implication de nouveaux acteurs, dès la phase de conception du projet, conduit à la confrontation de points de vue différents - voire contradictoires - à partir desquels se construit le consensus. Ce processus a-t-il été mis en œuvre dans le cas du PER Néouvielle et, si oui, en quoi a-t-il été porteur d'innovation?

\section{Un objectif d'innovation et d'excellence peu lisible dans le programme d'action}

Le projet de PER Néouvielle est principalement orienté vers la mobilisation de ressources patrimoniales, non délocalisables et intrinsèques au territoire. Le programme d'action, tel qu'affiché dans la convention-cadre État/Association pour la valorisation du massif du Néouvielle (2011) et en cours de réalisation en 2015, révèle cependant un déséquilibre majeur, car l'essentiel des investissements (83\%) est destiné à l'amélioration des quatre refuges existants et à la construction d'un cinquième à Aygues Cluses, en réponse à la demande des guides soucieux de réduire les distances entre la Glère et Orédon. Le soutien spécifique accordé par l'État au titre de la labellisation PER s'élève à $932500 €$, dont $812500 €$ seront dédiés exclusivement à l'amélioration de l'offre des refuges (action 4). Or, l'argument patrimonial est totalement absent de cette action qui aurait pourtant pu comporter un volet dédié à la valorisation de l'héritage pyrénéiste dans le massif. La valorisation du patrimoine, dont le contenu précis ne figure pas dans le dossier, contrairement aux autres opérations, ne bénéficie que de $1,9 \%$ du montant total des investissements. Le patrimoine, outil d' « innovation visant à l'excellence », n'a pas été, semble-t-il, mis en avant à la hauteur des objectifs de la politique PER.

L'affectation des trois-quarts des fonds à l'amélioration des refuges pose également des questions quant à la nature de l'opération. Les refuges, vétustes, accueillent actuellement 10400 nuitées pour une fréquentation totale de 200000 visiteurs/an aux portes de la réserve (parc national des Pyrénées, 2014). Obtenir une « augmentation de fréquentation de plus de 100000 visiteurs / an » (objectif PER) avec la seule construction de 20 à 40 lits supplémentaires en refuge, c'est-à-dire tout au plus 3000 nuitées/an supplémentaires ${ }^{8}$, est une

8 Calcul en hypothèse haute, établi en prolongeant les résultats de la fréquentation dans les refuges actuels. chimère. La rénovation des portes d'entrée et la réhabilitation d'un sentier de randonnée pour faciliter un accès aux personnes à mobilité réduite peuvent contribuer à atteindre cet objectif, mais seulement pour l'accueil de véhicules à la journée, donc en excursion : sans hébergement, pas de dépenses ni d'impact économique?

L'inégale répartition des fonds témoigne par ailleurs de la priorité accordée à l'équipement touristique du massif. Il s'agit, en effet, à travers la création ou la modernisation de refuges, de permettre la réalisation d'un vieux projet de « tour du Néouvielle », inspiré de circuits comparables existants dans d'autres massifs de montagne comme celui, relativement proche, des Encantats, sur le versant sud des Pyrénées. C'est, selon les dires d'un ancien agent de la de la direction départementale des territoires des Hautes-Pyrénées (DDT 65), autour de l'idée de « tour » que s'est construit le projet de PER :

"Très vite, c'est cette notion de "tour de massif" qui a émergé comme étant l'idée du produit touristique à proposer : il fallait travailler sur les refuges, il fallait travailler sur les entrées, sur les circuits, sur la signalisation, sur l'accompagnement, bref on était sur une vision très touristique, mais d'un produit touristique nouveau, qui n'était pas le tourisme de masse des stations de ski, mais qui s'adressait au tourisme nature, au tourisme de randonnée. Été, hiver. Composante émergente du tourisme d'hiver chez nous, mais non structurée : la pratique se fait par des initiatives de clubs sur des initiatives ponctuelles, mais elle n'est pas organisée en tant que produit commercialisé comme on pouvait voir sur les Encantats ou sur les sites de la Vanoise [...] » (Entretien réalisé le 12 février 2014 avec un ancien agent de la DDT 65).

Prétendument en rupture avec le tourisme de masse, ce projet se fixe néanmoins pour objectif l'accroissement de l'attractivité touristique du massif du Néouvielle, visant une augmentation de la fréquentation de plus de 100000 visiteurs/an.

Or, cette hausse de la fréquentation peut vite s'avérer problématique dans un massif où la question de la maîtrise des flux constitue une préoccupation ancienne. Dès 1996, le SIVU Aure-Néouvielle (4 communes) est d'ailleurs créé pour tenter d'y répondre en limitant l'accès à la réserve de Néouvielle par la route départementale 177 et en organisant l'accueil et le stationnement aux abords du lac d'Orédon. Le dernier plan de gestion de la réserve, élaboré par le parc national des Pyrénées, relève la forte affluence estivale qui, malgré une tendance

\footnotetext{
9 Les sciences économiques séparent les impacts du développement touristique en impact primaire, lié à de l'achat de biens et de services nécessaires à l'activité et aux dépenses effectuées par les visiteurs présents sur le territoire : skieurs, randonneurs, accompagnants... et en impact secondaire, la propagation de cette « injection initiale » de ressources dans l'économie locale, $\mathrm{du}$ fait des relations entre les agents économiques du territoire.
} 
à la baisse, demeure élevée et très concentrée spatialement. Il souligne également l'impact de la fréquentation hivernale, moindre, mais aux effets notables sur la biodiversité. Par conséquent, les objectifs de croissance des flux de visiteurs affichés par le PER apparaissent en contradiction flagrante avec ceux du plan de gestion qui visent à « limiter au mieux la fréquentation hivernale de façon à éviter le dérangement de la faune », en envisageant, dans certains secteurs, le maintien de mesures de contention de la fréquentation : ainsi, le site classé d'Estibère « doit être soustrait au mieux à la fréquentation touristique. La politique engagée en ce sens (absence volontaire de balisage et d'entretien des sentiers) devra être poursuivie et accentuée » (Parc national des Pyrénées, 2012). Pour les chargés de mission naturalistes du parc et les membres de son conseil scientifique, le marquage des sentiers ne suffira pas à canaliser les flux, le hors sentier étant très facile et très tentant dans le massif pour $y$ découvrir ses paysages cachés. Le parc national fait état de «son inquiétude » quant aux "dérangements probables » du Grand Tétras, espèce emblématique protégée du patrimoine faunistique des Pyrénées, dans ses aires de nidification, c'est-à-dire dans ses lieux de survie.

La diversification touristique et la transition d'une économie actuellement portée par les stations de ski vers un tourisme plus durable semblent hors d'atteinte avec ce programme PER. Comment en est-on arrivé là ? Les conditions d'élaboration du projet (« un projet de tourisme particulièrement respectueux $\mathrm{du}$ milieu naturel et complémentaire aux activités développées au sein des stations de montagne avoisinantes ») expliquent cette différence entre objectifs et réalisations.

\section{Des facteurs de blocage organisationnel local}

Le projet PER est né à l'initiative et grâce au concours de la direction départementale des territoires (DDT) des Hautes-Pyrénées. C'est ce service de l'État qui a permis de donner corps à $l^{\prime}$ « intention » de l'élue présidente de l'association pour la valorisation du massif du Néouvielle, qui « avait plein d'idées sur ce site, voulait faire des choses sur le site, mais les choses n'étaient pas exprimées, elles n'étaient pas concrètes ; elle avait une intention, mais pas une vision " (Entretien réalisé, à Tarbes, le 12 février 2014 avec un ancien agent de la DDT 65 en charge de la zone montagne). La DDT pallie l'absence de vision stratégique d'une élue ne parvenant pas à assumer pleinement son leadership politique et assure la concrétisation du projet en s'inspirant d'une idée ancienne, formulée en 1985, par la direction départementale de l'Équipement et le service d'études et d'aménagement de la montagne qui avaient souhaité créer un réseau d'auberges d'altitude dans le Néouvielle. Les dissensions entre élus avaient alors eu raison du projet. Or, les acteurs locaux, nombreux (parc national, conseil général, maires des communes accueillant les quatre stations de ski et la réserve régionale voisine d'Aulon, élus des villages dotés d'un riche patrimoine bâti en vallée d'Aure réunis dans un Pays d'art et d'histoire, guides, propriétaires des refuges) n'ont pratiquement pas changé depuis cette date. La DDT intervient ainsi, dans un contexte difficile, pour créer du lien et impulser une dynamique.

Conçu et pour partie rédigé par l'État, le projet du PER Néouvielle est alors recyclé dans une programmation touristique en panne depuis longtemps. Le PER est perçu comme un moyen de lever des fonds pour mener à bien, au minimum, une rénovation de refuges. Il fait également office de « labellisation compensatoire » (Clarimont et Bessière, 2016). En effet, l'Association pour la valorisation du Néouvielle avait été créée, en 2007, afin de faire inscrire le site dans le programme régional "Grands sites Midi-Pyrénées ». Son initiative échoue, quatre autres dossiers étant incontournables dans les Hautes-Pyrénées (Lourdes, Gavarnie, le pic du Midi et Cauterets). Le PER offre l'occasion de relancer un projet avorté. Certes, le cas du Néouvielle n'est pas exceptionnel, ce type de dispositif servant souvent de relais pour des opérations déjà envisagées, mais non encore réalisées, faute de moyens. Pour autant, il est important d'examiner les conditions qui permettent à la société locale de s'exonérer des objectifs d'une politique pour en réaliser d'autres, car ces bifurcations déterminent la capacité locale à atteindre les objectifs affichés d'innovation et de transition touristique.

Contre-pied d'un label " Grand site Midi-Pyrénées » non obtenu, le premier dossier de PER déposé fonde un argumentaire sur l'existence d'un bassin d'attractivité touristique déjà en place, sur la (re)valorisation d'un capital de notoriété et la préservation d'éléments patrimoniaux remarquables. La Datar ne retient pas cette première candidature en 2010, car le parc national des Pyrénées, qui gère pourtant la réserve naturelle nationale du Néouvielle, n'a pas été consulté.

\section{Un modèle cloisonné, une grande complicité entre des acteurs établis}

Éloigné des «PER-étendards » qui portent à un niveau plus prospectif le projet du territoire et contribuent à une recomposition des systèmes locaux d'action territoriale (Milian et Bacconnier-Baylet, 2014), le PER Néouvielle est construit par des acteurs différents qui se saisissent tous des éléments de projet à leur disposition pour construire une politique de développement : la complexité de la construction politique de ces dispositifs repose sur des relations de pouvoir parallèles entre élus et services de l'État. Le schéma est classique et a été décrit dès 1966 par Jean-Pierre Worms. Porté par l'État et quelques élus influents du territoire, il est marqué par le caractère descendant de la démarche (de l'État vers la conseillère départementale et l'association qu'elle préside). Cette construction marque la réactivation, par 
certains services de l'État, d'un ancien schéma aménagiste du massif (reprenant, sous une forme plus acceptable, car reposant sur les cheminements, l'idée d'extension des stations). Nous sommes ici typiquement dans le schéma classique des relations de complicité du «préfet et ses notables » où les acteurs s'entendent pour s'adapter au passage de la politique de zonage à la politique de compétitivité. Utiliser l'Administration comme agent de changement est problématique, avait déjà démontré Pierre Grémion en 1976 : le manque d'appropriation du projet par la population locale et même par une large partie de ses élus, le faible niveau de connaissance des habitants et des acteurs locaux de ce dispositif attestent de la récupération de l'argument patrimonial au profit d'une procédure « top-down » déconnectée de la mise en projet locale. Si dans certains PER portés par des entreprises ou des associations, l'hybridation public/privé a pu s'avérer féconde et porteuse d'innovation (Lardon et al., 2014), dans le cas du Néouvielle, cela n'a pas enclenché de dynamiques nouvelles et le pôle n'est pas devenu un lieu de débat territorial. La construction d'un projet dans une approche « bottom-up » et la mise en place de nouvelles formes de gouvernance territoriale (débattues et fondées sur la qualité) ont fait défaut. On remarquera également que les modes d'implication des populations dans l'opération apparaissent flous lors des entretiens. Les acteurs impliqués semblent avoir vu dans ce projet une occasion de « reprendre la main » : l'État contrôlerait à nouveau le développement territorial ; le conseil général resterait présent et les maîtres d'ouvrage des opérations (Club alpin français, commune de Saint-Lary, Parc national, ASPTT) bénéficieraient de crédits pour moderniser les équipements obsolètes. En quête d'une image plus "nature ", respectable, en recherche de possibilités d'étendre leur offre de ski à ses marges et de légitimer des pratiques hybrides associant toutes les formes de glisse (du ski de piste et du snowboard à la découverte de la nature en ski de randonnée), les stations de ski alpin bénéficient aussi de la rhétorique patrimoniale développée par le pôle d'excellence rurale. En ce sens, ce projet apparaît, par l'effet que lui confère le label, un instrument de marketing territorial qui rejoint d'autres formes de valorisation territoriale (autres PER, politiques des grands sites régionaux ou de France, contrats de pôle touristique...).

La coalition entre services de l'État et collectivités permet à chaque acteur d'affirmer sa présence et son rôle au sein de sa sphère $d^{\prime}$ action dans le portage $d u$ projet. Nous retrouvons ici un des schémas classiques $\mathrm{du}$ fonctionnement du système politico-administratif local qui explique en grande partie l'échec du changement et des processus innovants (Crozier, 1963). En effet, la dynamique des processus d'innovation repose sur les échanges d'information, d'expériences, les opportunités de trouver des compétences diversifiées et de multiplier les canaux de transmission entre des partenaires très ouverts (Bouneau et Lung, 2014). Or, pour des raisons de posture politique locale (liens entre élus et représentants associatifs), les acteurs n'ont pas ouvert la conception du projet à d'autres partenaires, mêmes proches (observatoire, communes des vallées, associations locales du patrimoine, lesquels ont été volontairement ignorés) (fig. 2). Ils se sont ainsi trouvés dans l'impossibilité de fédérer des capacités et des processus nouveaux extérieurs au système local, d'initier l'interaction, la porosité, le partenariat, l'échange de connaissances et d'expériences avec des organisations pouvant apporter des compétences nouvelles et diversifiées. Ainsi, la commune d'Aulon, limitrophe, qui mène depuis des décennies une politique innovante de tourisme durable (installation d'hébergements ruraux contrôlés par la population, installation de jeunes agriculteurs, $\mathrm{d}^{\prime}$ artisans par une nouvelle génération d'élus issus de la mouvance autogestionnaire des années 1970) n'a guère été associée au dossier en raison de divergence politique. La vallée $\mathrm{d}^{\prime}$ Aure, bien que d'une autre mouvance, et dont le label " Pays d'art et d'histoire ", décerné par le ministère de la Culture, témoignait pourtant d'une volonté de valorisation patrimoniale, a quant à elle été écartée du projet pour des raisons identiques. Ces ressources, ce potentiel présent sur le territoire n'ont pas pu être activés parce qu'ils ne sont pas séparables des postures politiques individuelles et des organisations qui en sont dépositaires. Le processus complexe de partage des savoirs, des savoirfaire et des expériences que supposent les dynamiques d'innovation n'a pas pu être initié du fait de l'impossible interaction entre des acteurs mobilisant des registres d'échange différents.

La procédure de Pôle d'excellence rurale n'a pu être appliquée que dans la mesure où les acteurs locaux ont pu en « plier » les attendus afin d'y faire entrer leur rationalité propre, même éloignée de l'innovation. Les pratiques « augmentées » de l'activité touristique proposée par les aménagements du PER (refuges, portes d'entrée qui ouvrent aux touristes la traversée peu contrôlée de grands espaces naturels fragiles), le pouvoir $\mathrm{d}^{\prime}$ « engendrement », $d^{\prime}$ « innovation », de diversification requis par le recours au PER n'a pas eu lieu. La différence entre les objectifs de valorisation patrimoniale affichés et les opérations de rénovation de lits de refuge finalement programmées repose sur l'impossibilité de créer des opportunités de trouver des compétences et des ressources diversifiées. Si l'innovation est absente du dossier, c'est donc aussi parce que le projet s'est contenté de recycler rapidement de vieux dossiers en souffrance, sans prendre en compte les apports nouveaux à mobiliser au 


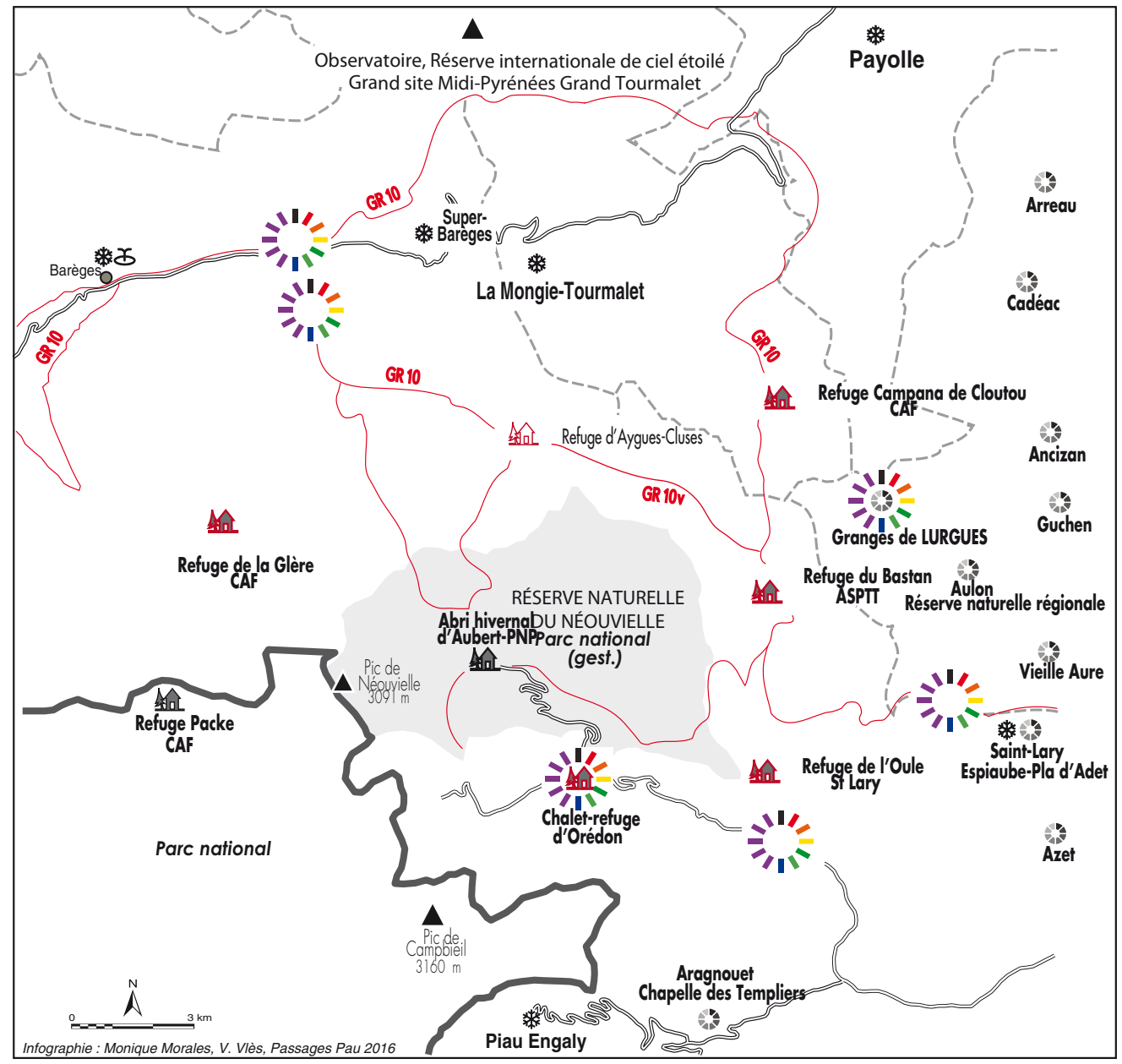

\begin{tabular}{|lll|}
\hline Limite de la Réserve Naturelle du Néouvielle & \\
Limite du Parc National des Pyrénées & Station de ski & \\
Station thermale & Itinéraire & Porte d'entrée PER \\
\hline--- Limite de canton & Routes départementales & \\
\hline \hline
\end{tabular}

Fig. 2. Acteurs et ressources patrimoniales du Néouvielle : stations de ski, patrimoine naturel, patrimoine architectural et grand paysage (cartographie : V. Vlès, 2016).

sein des ressources patrimoniales agricoles, pastorales, artisanales, industrielles (hydroélectricité) ou architecturales du massif.

\section{Des opérations bien banales}

Seule action " patrimoniale », une opération prévue par le PER consiste à accueillir dans un bâtiment en altitude (accessible aux visiteurs) un point de vente de fromages. Le problème est que le Néouvielle n'est pas un territoire de production fromagère. Les cheptels y sont élevés pour l'embouche. L'opération revient à « importer » en altitude, par transport motorisé (donc avec émission de gaz à effet de serre), des produits alimentaires fabriqués ailleurs.
De même, force est de constater que les effets d'entraînements de dynamique territoriale pour sortir des situations de tourisme sectoriel (ski alpin) n'ont pas rencontré pour l'heure le succès escompté. Cela s'explique, sur le plan local, par la faible connaissance et le peu d'appropriations du dispositif, par la mise à l'écart du tissu socioéconomique et l'absence de projets structurants et innovants basés sur les ressources patrimoniales du territoire. Annoncé comme un levier de diversification territoriale raisonné, le dispositif PER se révèle être davantage un outil d'accueil de visiteurs. Le PER Néouvielle, s'il favorise la restauration et la création de cinq refuges, de six portes d'entrée supposées favoriser l'augmentation de la fréquentation, la création de services commerciaux en altitude (dans un secteur où le parc national 
pourrait s'y opposer), n'a pas réussi à porter un projet collectif innovant de diversificationéconomique. Cela complique fortement et rend peu probable l'atteinte des objectifs affichés.

\section{Innovation et patrimoine : une rhétorique dépourvue d'effets?}

Pour innover, les acteurs du Néouvielle auraient pu considérer la valorisation des ressources locales comme faisant partie intégrante du processus de développement durable. Il s'agissait d'abord de repérer si les conditions permettant de promouvoir un panier de biens et de services territorialisés étaient réunies : quels sont les produits et les services potentiellement et réellement constitutifs du « panier typique Néouvielle ${ }^{10}$ »? Quel(s) en est (sont) le(s) produit(s) leader? Quelles sont les dynamiques territoriales de valorisation déjà existantes de ces produits ? Dans un projet de réflexion sur la mise en valeur concertée des ressources locales autour du massif du Néouvielle, quels acteurs doivent être associés ? Quel est le périmètre territorial pertinent? Créer des services et des biens propres, spécifiques au lieu, notamment dans les domaines agroalimentaire et pastoral, aurait pu permettre d'élaborer un produit phare, un élément de référence typique nécessaire à l'élaboration et au lancement d'un panier de biens et de services à valoriser. Pour l'heure, les produits labellisés commercialisés dans le Néouvielle sont communs à l'ensemble des régions montagneuses, et pas seulement aux Pyrénées. L'absence d'un réseau de vente en circuit court local complique encore la communication sur ces produits. Sur le plan gastronomique ou alimentaire, les plats et les diverses traditions ont été oubliés. Même l'image touristique du Néouvielle n'est pas ou peu mise en avant et tous les produits phares actuels du territoire sont ceux de ses zones périphériques : il existe un riche patrimoine architectural (art roman) dans les vallées voisines, mais il n'est guère mentionné par le programme PER. On découvre dans les restaurants et les commerces du Néouvielle des produits tels que la garbure béarnaise, le haricot tarbais, les vins du Jurançon, les produits basques ou encore le porc noir gascon. La restauration y est certes essentiellement composée de plats "montagnards ", mais d'une autre montagne, notamment des Alpes (tartiflette, raclette, reblochon). Le Néouvielle, à l'heure actuelle, ne présente

\footnotetext{
10 Les années 1990 ont été marquées par la crise des systèmes agricoles intensifs, mais aussi par un regain d'intérêt pour la qualité dans la production agroalimentaire et les services rendus par l'agriculture. Dans les zones rurales en difficulté économique, la valorisation par le tourisme de ces ressources nouvelles a été le moteur de stratégies alternatives de développement. De l'analyse de ces stratégies, menée depuis près de 15 ans sur différents territoires est né le concept de panier de biens et de services territorialisés (Pecqueur 2001 ; Mollard et Pecqueur, 2007).
}

aucune action qui pourrait devenir un élément du « panier de biens et services » (Pecqueur, 2001), c'est-àdire ayant un impact économique primaire et secondaire positif pour le territoire (Maurence, 2012).

\section{Conclusion}

Invoquer n'est pas mobiliser. Le dispositif PER Néouvielle semble avoir été le lieu d'un processus $\mathrm{d}^{\prime}$ « habillage patrimonial ». Fort d'une dimension sacralisante aux yeux de la société (locale et extralocale), l'argument patrimonial participe du processus de légitimation des formes de gouvernance locale. Le projet $\mathrm{d}^{\prime}$ « excellence » a été récupéré pour servir d'alibi à des actions fort éloignées du processus d'innovation. Elles visent prioritairement à la poursuite et à la prise en charge d'opérations que le productivisme touristique des stations n'avait pas réussi à mener à bien jusqu'à présent. Au lieu de modifier le fonctionnement initial du système politico-administratif de gouvernance locale du tourisme et d'impulser une nouvelle organisation interne porteuse d'innovation, cette procédure d'excellence et de compétitivité des territoires a plutôt reproduit, dans le Néouvielle, un ordre traditionnel fait de coopérations, provoquant peu de perturbations et suscitant de faibles réactions.

L'expérience de diversification semble avoir fonctionné selon un référentiel des années 1980 non modifié dans ses fondements ou dans sa mécanique. La procédure $\mathrm{d}^{\prime}$ « innovation compétitive » a reproduit les interventions et la position des acteurs dans un ensemble assez bien structuré et dont la stabilité est assurée par la permanence des décideurs et leurs rapports privilégiés avec l'Administration. Cette régulation croisée a freiné le changement et confirmé les choix d'un système touristique résilient : le projet n'est pas parvenu à fonder une solidarité de gestion territoriale du tourisme entre les stations et leur arrière-pays ${ }^{11}$, écartant ainsi d'emblée la construction d'une aire touristique plus large permettant de diversifier les produits et de renouveler les clientèles du massif. Dans ce processus, l'immatériel aurait pu devenir un levier majeur : les stations de ski ne seraient plus seulement les unités socioterritoriales du "petit », $\mathrm{du}$ « proche » et de $\mathrm{l}^{\prime}$ « intense » (Pecqueur et al., 2009), caractérisées par des communautés d'habitants, de touristes et d'entreprises, elles auraient été contraintes de

\footnotetext{
11 L'aire touristique peut être approchée comme le territoire économique, social et politique marqué par des relations soutenues entre une station et son arrière-pays : elle est marquée par l'ensemble des liens tissés entre la station de tourisme, son territoire originel et son espace de déploiement désormais élargi et transformé (Vlès, 2015). Cette extension territoriale permet une diversification et une innovation de produits.
} 
s'ouvrir sur une « aire touristique » plurifonctionnelle, plus large, diversifiée, tant dans ses éléments productifs que dans ses logiques habitantes. Processus légitimant, la patrimonialisation annoncée de ressources matérielles (patrimoines bâtis) comme immatérielles (patrimoine paysager, patrimoine alimentaire, savoir-faire, agropastoralisme), a certes apporté une garantie symbolique aux yeux des acteurs et a abouti à une démarche conventionnée de modernisation d'un parc d'hébergement de pleine nature, mais n'a rien changé à l'état d'ouverture de la société locale ni au jeu des stratégies d'acteurs. Elle n'a pas enclenché un processus de transformation globale autour des questions de durabilité, d'adaptation au changement climatique, encore moins de transition touristique. Paradoxalement, la position de l'État aujourd'hui plus éloignée des lieux de la décision n'a pas aidé à renouveler les formes de gouvernance locale. L' « utilisation » et l'inscription purement rhétorique du patrimoine dans le projet de PER témoignent de l'attachement des acteurs de ce territoire à des représentations liées à la tradition, au passé et à l'héritage, inhibant, au moins dans les perceptions, toute dimension innovatrice.

\section{Références}

Alvergne C., De Roo P., 2008. Les pôles d'excellence rurale: regards sur une politique française en direction de l'espace rural, Organisations et Territoires, 17, 1, 73-78.

Alter N., 2000. L'innovation ordinaire, Paris, Presses universitaires de France.

Association pour la valorisation du massif du Néouvielle, 2010. Néouvielle, destination nature. Dossier de candidature Pôle d'excellence rurale 2010.

Atout France, 2013. Panorama du tourisme de la montagne. Cahier $n^{\circ} 2$ : emploi et retombées économiques, Paris, Atout France.

Barral F., Simoulin V., Thumerel B., 2010. Ingénierie de projet et excellence territoriale, Territoires 2040, 2, 137-148.

Barthe L., Milian J., Taulelle F. (Eds), 2009. Les pôles d'excellence rurale : processus, gouvernance et plus-value dans les trajectoires de développement des territoires. Rapport pour la Délégation interministérielle à l'Aménagement et à la Compétitivité des Territoires (DIACT), Toulouse, Université de ToulouseLe Mirail.

Bessière J., 2001. Valorisation du patrimoine gastronomique et dynamiques de développement territorial. Le haut plateau de l'Aubrac, le pays de Roquefort et le Périgord noir, Paris, L'Harmattan.

Bessière J. (Ed.), 2012. Innovation et patrimoine alimentaire en espace rural, Versailles, Quæ.

Bouneau C., Lung Y. (Eds), 2014. Les trajectoires de l'innovation. Espaces et dynamiques de la complexité (XIX $\mathrm{X}^{e} \mathrm{XX} \mathrm{I}^{e}$ siècles), Bruxelles, PIE-Peter Lang.

Bourdeau P., 2009. De l'après-ski à l'après-tourisme, une figure de transition pour les Alpes? Réflexions à partir du cas français, Journal of Alpine Research/Revue de géographie alpine, http://rga.revues.org/1049.
Clarimont S., Bessière J., 2016. L'adaptation au changement. « Néouvielle, destination nature » ou la mobilisation de la ressource patrimoniale comme diversification touristique, in Vlès V., Bouneau C. (Eds), Stations en tension, Bruxelles, PIE-Peter Lang, 43-56.

Clarimont S., Vlès V., 2016. Les contestations sociales du développement touristique dans les Hautes-Pyrénées : le rendezvous manqué de l'innovation territoriale?, Journal of Alpine Research/Revue de géographie alpine, http://rga.revues.org/ 3218.

Clarimont S., Vlès V. (Eds), 2008. Tourisme durable en montagne : entre discours et pratiques, La Plaine Saint-Denis, AFNOR.

Convention cadre État/Association pour la valorisation du massif du Néouvielle, PER nD065814, Vielle-Aure, 14 mars 2011.

Crozier M., 1963. Le phénomène bureaucratique. Essai sur les tendances bureaucratiques des systèmes d'organisation modernes et sur leurs relations en France avec le système social et culturel, Paris, Seuil.

Doloreux D., Filion P., Klein J.-L., 2005. Systèmes régionaux et innovation : le cas de la Beauce québécoise, in Guillaume R. (Ed.), Globalisation, systèmes productifs et dynamiques territoriales. Regards croisés au Québec et dans le Sud-Ouest français, Paris, L'Harmattan, 215-237.

Epstein R., 2009. Après la territorialisation : le gouvernement à distance, in Vanier M. (Ed.), Territoires, territorialité, territorialisation. Controverses et perspectives, Rennes, Presses universitaires de Rennes, 131-139.

George-Marcelpoil E., 2007. Réflexions sur l'ancrage territorial des stations de montagne, in Bourdeau P. (Ed.), Les sports d'hiver en mutation. Crise ou révolution géoculturelle, Paris, Hermès science publications/Lavoisier, 161-172.

Grémion P., 1976. Le pouvoir périphérique: bureaucrates et notables dans le système politique français, Paris, Seuil.

Landel P.-A., Sénil N., 2008. Les nouveaux territoires et leurs noms entre projet et compétitivité: le cas des «pôles d'excellence rurale », L'espace politique, doi: 10.4000/espacepolitique.270.

Lardon S., Chia E., Rey-Valette H., 2008. Introduction : Dispositifs et outils de gouvernance territoriale, Norois, 209, doi: $10.4000 /$ norois.2602.

Lardon S., Milian J., Loudiyi S., LeBlanc P., Barthe L., Taulelle F., 2014. Du potentiel à l'action : la gouvernance territoriale des pôles d'excellence rurale, Norois, 233, doi: 10.4000/ norois.5380.

Le Galès P., 2013. La gouvernance territoriale sous pression de la crise et de la restructuration de l'État, in Pasquier R., Simoulin V., Weisbein J. (Eds), La gouvernance territoriale. Pratiques, discours et théories, Paris, LGDJ, 289-300.

Leloup F., Moyart L., Pecqueur B., 2005. La gouvernance territoriale comme nouveau mode de coordination territoriale ?, Géographie Économie Société, 7, 4, 321-331.

Maurence E., 2012. La mesure de l'impact économique d'un événement touristique. Rapport d'étude, Paris, ministère de l'Économie, des Finances et de l'Industrie, DGCIS, Sousdirection de la Prospective, des Études économiques et de l'Évaluation.

Micoud A., 2004. Des patrimoines aux territoires durables. Ethnologie et écologie dans les campagnes françaises, Ethnologie française, 34, 1, 13-22. 
Milian J., Bacconnier-Baylet S., 2014. Requalifier les territoires de l'action locale : l'exemple des pôles d'excellence rurale de la filière bois, Territoire en mouvement, 22, 54-67.

Ministère de l'Espace rural et de l'Aménagement du territoire/ ministère de l'Alimentation, de l'Agriculture et de la Pêche, 2010. Pôles d'excellence rurale 2010. Cahier des charges de l'appel à projets, http://poles-excellence-rurale.datar.gouv.fr/sites/ poles-excellence-rurale.datar.gouv.fr/files/cahier $\% 20 \mathrm{des} \%$ 20charges\%20PER2G\%202V\%2016avril2010.pdf.

Mollard A., Pecqueur B., 2007. De l'hypothèse au modèle du panier de biens et de services. Histoire succincte d'une recherche, Économie rurale, 300, 110-114.

Parc national des Pyrénées, 2012. Étude quantitative de la fréquentation du PNP et de la réserve nationale du Néouvielle du 15 juin au 15 septembre 2012, Tarbes, Parc National des Pyrénées.

Parc national des Pyrénées, 2014. Étude des fréquentations estivales, focus des sites d'accueil touristiques, Tarbes, Conseil général des Hautes-Pyrénées.

Pecqueur B., 2001. Qualité et développement territorial: l'hypothèse du panier de biens et de services territorialisés, Économie rurale, 261, 1, 37-49.

Pecqueur B., Senil N., Canova N., Robinet N., Julian X., 2009. La place du patrimoine et de la culture dans les projets de développement territorial. Rapport pour la Délégation interministérielle à l'Aménagement et à la Compétitivité des Territoires
(DIACT), http:/ / zonages.territoires.gouv.fr/sites/default/ files/datar/2009-per-place-du-pratimoine-cermosem.pdf.

PER (Pôles d'excellence rurale), 2014. Pôles d'excellence rurale : http:/ / poles-excellence-rurale.datar.gouv.fr/les-poles-dexcellence-rurale.

Prats M., Charry J.-C., Glass B., 2000. Contribution du tourisme au développement durable de la moyenne montagne. $4^{\mathrm{e}}$ partie : Un plan d'actions pour le tourisme en «moyenne montagne ", Paris, la Documentation française, http:// www.ladocumentationfrancaise.fr/var/storage/rapportspublics/014000281.pdf.

Siné A., 2001. Responsabilité politique : de la fin du modèle weberien aux nouvelles figures de légitimation de l'action publique, Politiques et management public, 19, 2, 45-62.

Vlès V., 2014. Métastations. Mutations urbaines des stations de montagne. Un regard pyrénéen, Pessac, Presses universitaires de Bordeaux.

Vlès V. (Ed.), 2015. TRATSO-2012/2015. Les trajectoires des aires touristiques dans le grand Sud-Ouest français depuis le XIX siècle : dynamiques d'innovation, mises en tension et enjeux prospectifs. Bilan de la recherche financée dans le cadre de l'appel à projet du Conseil régional d'Aquitaine 2012, Pessac, Maison des Sciences de l'Homme d'Aquitaine, https://hal.archivesouvertes.fr/hal-01275309.

Worms J.-P., 1966. Le préfet et ses notables, Sociologie du travail, $8,3,249-275$.

Reçu le 16 juin 2015. Accepté le 13 septembre 2016. 